\title{
Tratamento dos Leiomiomas por Embolização das Artérias Uterinas
}

Uterine artery embolization in the treatment of uterine leiomyomas

Marcos de Lorenzo Messina, Valéria Márcia Martins, Ayrton Cássio Fratezi, Hans Wolfgang Halbe, Nilo Bozzini, Sérgio Conti Ribeiro, José Aristodemo Pinotti

\section{RESLMO}

\begin{abstract}
Objetivos: avaliar os resultados da embolização das artérias uterinas (EAU) como tratamento do leiomioma uterino.

Métodos: foram estudadas 18 mulheres com diagnóstico ultra-sonográfico de leiomioma uterino submetidas à EAU com partículas de álcool polivinílico (PVA). O acesso arterial foi realizado por punção da artéria femoral e a progressão do cateter foi monitorada até atingir seletivamente as artérias uterinas. A avaliação dos resultados foi realizada pelo estudo ultrasonográfico antes e três meses após o procedimento. Foi realizado controle clínico evolutivo para avaliação das características menstruais e do volume uterino.

Resultados: houve insucesso técnico em três casos por dificuldade de cateterização das artérias uterinas. $O$ sangramento e a dismenorréia foram controlados no intervalo de três meses em 86 e 60\% das pacientes, respectivamente. A média dos volumes uterinos iniciais foi de $381 \mathrm{~cm}^{3}$ e após 3 meses foi de $263 \mathrm{~cm}^{3}$. Houve redução média de 27,4\% no volume uterino três meses após o tratamento. Três pacientes tiveram insuficiência ovariana clínica e laboratorial ( $20 \%$ dos casos).

Conclusões: a EAU representa alternativa terapêutica para o tratamento dos leiomiomas uterinos. Em virtude do risco de insuficiencia ovariana esse procedimento é reservado para pacientes com mais de 45 anos de idade ou com prole completa.
\end{abstract}

PALAVRAS-CHAVE: Leiomioma uterino. Embolização das artérias uterinas. Falência ovariana.

\section{Introdução}

O leiomioma uterino é a neoplasia benigna mais freqüente do aparelho genital feminino, acometendo até $40 \%$ das mulheres no menacme ${ }^{1}$. O seu tratamento clínico compreende diferentes tipos de medicações hormonais, sem haver medicamento capaz de erradicar o processo patológico. O emprego de análogos do GnRH é a alternativa mais eficiente para redução temporária do volume tumoral e dos sintomas clínicos, havendo o

Clínica Ginecológica do Departamento de Obstetrícia e Ginecologia do Hospital das Clínicas da FMUSP; Setor de Radiologia Vascular Intervencionista do Departamento de Radiologia do Hospital das Clínicas da FMUSP. Correspondência:

Marcos de Lorenzo Messina

Alameda Itú, 745/41

01421-000 - São Paulo - SP

e-mail: mmessina@uol.com.br

Tel.: (11) 3168-5311 inconveniente dos efeitos colaterais que impedem a sua utilização por tempo prolongado ${ }^{1}$.

Por outro lado, o tratamento cirúrgico dos leiomiomas varia, entre outros condicionantes, de acordo com o desejo reprodutivo da paciente. A miomectomia é a cirurgia clássica para remoção dos leiomiomas em pacientes que manifestam interesse de preservar o útero para gestações futuras ou simplesmente pelo desejo de manutenção do fluxo menstrual. De acordo com o número, localização e tamanho dos nódulos a cirurgia pode ser realizada por via histeroscópica, laparoscópica ou por laparotomia ${ }^{2}$. Ao contrário, quando a paciente não refere interesse reprodutivo futuro, a histerectomia é o tratamento de escolha dos leiomiomas. Entretanto, a histerectomia pode ser acompanhada de alterações da sexualidade por razões biológicas e psicossociais, sendo necessária discussão detalhada dos aspectos emocionais envolvidos na cirurgia por ocasião da proposta terapêutica ${ }^{3}$. 
Com objetivo de reduzir o tamanho dos nódulos de leiomioma a fim de possibilitar acesso cirúrgico menos invasivo, Ravina et al. ${ }^{4}$ em 1995 descreveram a embolização das artérias uterinas (EAU) como alternativa neoadjuvante no tratamento cirúrgico dos leiomiomas. A melhora da sintomatologia observada após o procedimento foi tão significativa que a EAU passou a ser considerada como nova opção terapêutica no tratamento do leiomioma uterino em mulheres que não queriam o tratamento cirúrgico convencional.

Até o presente momento cerca de 6000 pacientes com leiomiomas uterinos foram tratadas mediante EAU, verificando-se o controle da sintomatologia em $85 \%$ dos casos e redução média de $60 \%$ no volume uterino ${ }^{4-8}$.

No presente trabalho, são relatados os resultados iniciais de um serviço no tratamento de mulheres com leiomiomas uterinos que foram submetidas à EAU.

\section{Pacientes e Métodos}

Foram selecionadas, prospectivamente, 18 mulheres no menacme com diagnóstico clínico e ultra-sonográfico de leiomioma uterino, encaminhadas ao Ambulatório de Ginecologia do Hospital das Clínicas da FMUSP no período de 01 de março a 30 de setembro de 2000.

Pacientes com idade inferior a 35 anos, com desejo de gestação futura ou com antecedente de alergia a contraste iodado foram excluídas do estudo. Além disso, foram excluídas as pacientes com leiomiomas subserosos pediculados ou com alterações significativas na propedêutica cervical para rastreamento de neoplasias do colo uterino (colpocitologia oncológica e colposcopia). A média de idade das pacientes submetidas ao procedimento foi de 39,7 anos. As mulheres na faixa etária de 35 a 40,40 a 45 e 45 a 50 anos correspondiam respectivamente a $22 \%, 33 \%$ e $45 \%$ do total das pacientes.

Obteve-se consentimento pós-informado de todas as pacientes, elaborado de acordo com a Comissão de Ética do Hospital das Clínicas da Faculdade de Medicina da Universidade de São Paulo.

Foram realizadas dosagens de FSH, LH e estradiol entre o quinto e sétimo dias do ciclo menstrual para avaliação da reserva ovariana.

$\mathrm{Na}$ véspera do procedimento procedeu-se a nova avaliação ultra-sonográfica, sempre com o mesmo operador, utilizando aparelho da marca Toshiba, modelo Tosbee, com sonda endovaginal com transdutor de $5 \mathrm{MHz}$. O exame foi realizado na primeira fase do ciclo menstrual nas pacientes com ciclos regulares. Em todos casos a avaliação do endométrio foi compatível com a fase do ciclo mens- trual. As pacientes com ciclos irregulares ou mesmo em amenorréia foram orientadas a realizar o procedimento após o teste de gravidez negativo.

\section{Técnica de embolização}

Os procedimentos foram realizados no Setor de Radiologia Vascular Intervencionista - Instituto de Radiologia do Hospital das Clínicas da FMUSP - utilizando-se um aparelho Angiostar (Siemens), tendo as pacientes sido submetidas à sedação ou bloqueio peridural.

O procedimento foi realizado pela punção bilateral das artérias femurais comuns por meio da técnica descrita por Seldinger ${ }^{9}$, seguida de cateterismo seletivo das artérias hipogástricas com posterior realização de arteriografia diagnóstica para identificação do útero e da anatomia das artérias pélvicas. Os materiais utilizados foram: fios guias hidrofílicos, introdutores valvulados número 6 e cateteres cobra número 2 com diâmetro de $5 \mathrm{~F}$.

Após a análise da anatomia das artérias uterinas, realizou-se o cateterismo destas artérias e também uma nova arteriografia para identificação e estudo do útero e dos nódulos leiomiomatosos (Figuras 1A e 1B).

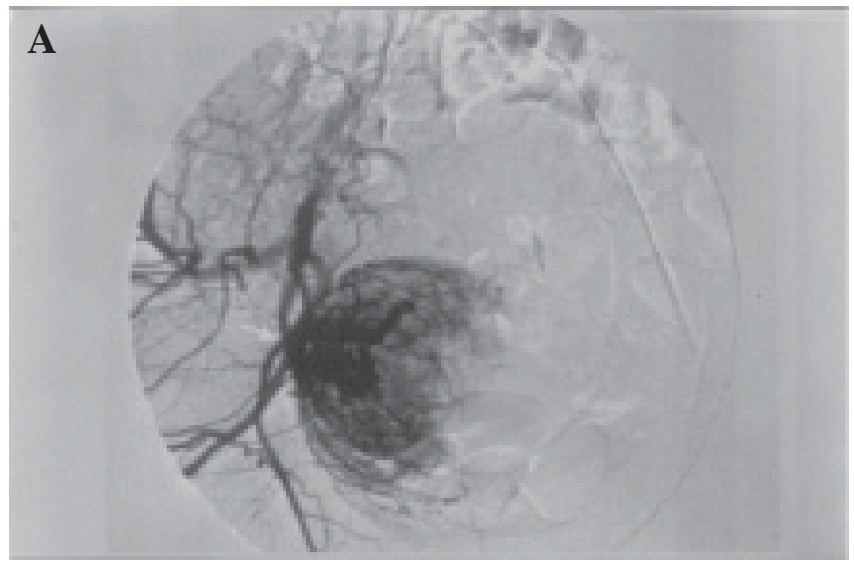

B

Figuras 1 - Artérias uterinas direita (A) e esquerda (B). As setas indicam a origem das artérias uterinas. Notar nesta arteriografia a presença de nódulo único, intensamente vascularizado nutrido igualmente pelas 2 artérias. 
Procedeu-se à embolização seletiva da artéria uterina relacionada ao leiomioma, utilizando partículas de PVA de 500 a 700 micra de diâmetro, até a total interrupção do fluxo sanguíneo pela artéria uterina. A quantidade de álcool polivinílico (PVA) empregada é dependente do volume uterino e da quantidade de leiomiomas existentes (Figu$\operatorname{ras} 2 \mathrm{~A}$ e $2 \mathrm{~B})$.
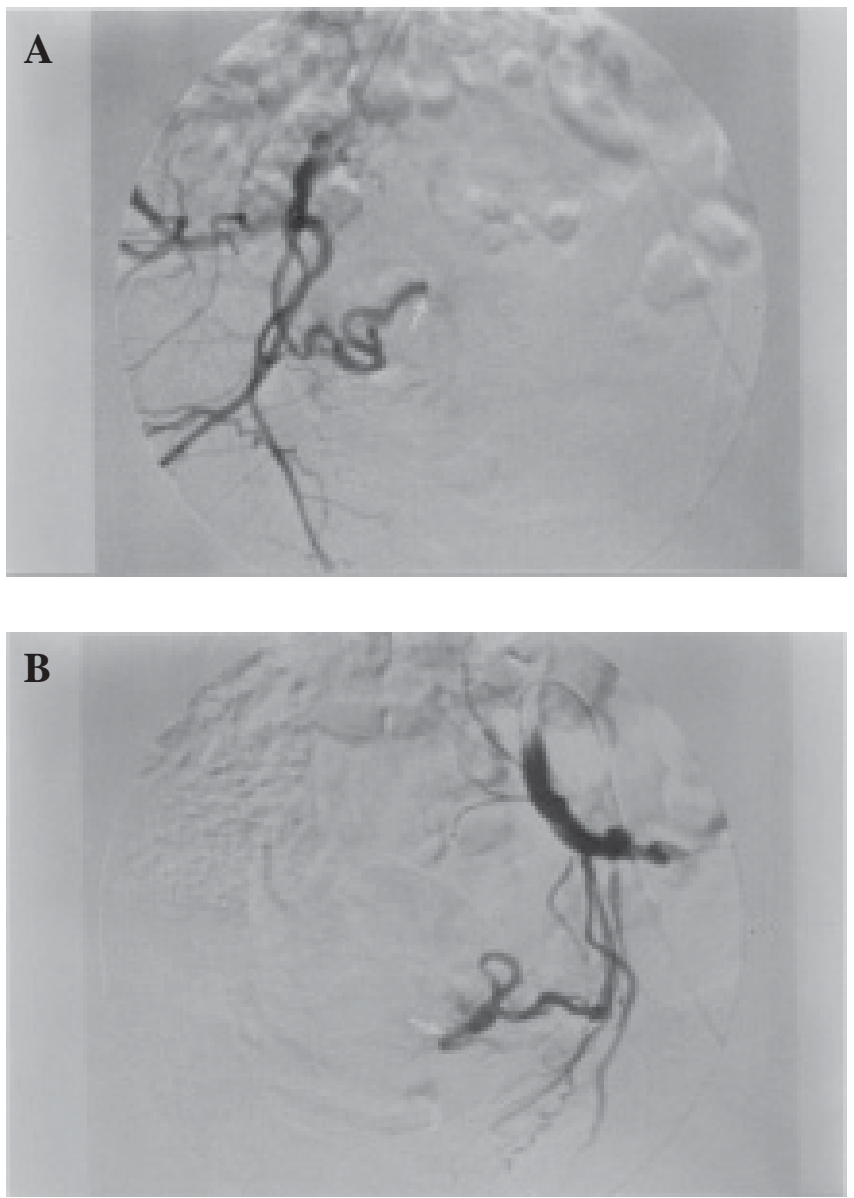

Figuras 2 - Artérias uterinas direita (A) e esquerda após embolização (B). As setas indicam as artérias uterinas com ausência total de fluxo sangüíneo. $O$ nódulo de mioma fica totalmente desvascularizado.

Após a realização do procedimento, as pacientes foram encaminhadas para a sala de recuperação, com monitoração contínua dos parâmetros vitais, retornando após sua estabilização para a enfermaria.

No período pós-operatório, de acordo com a intensidade da dor, utilizou-se paracetamol, codeína, meperidina ou antiinflamatório nãohormonal. As pacientes receberam alta hospitalar quando a dor era controlada satisfatoriamente com medicação por via oral. O seguimento das pacientes foi semanal no primeiro mês e, a seguir, mensal.

Os parâmetros avaliados nesta análise foram: volume uterino por meio de medidas seria- das por ultra-sonografia na primeira fase do ciclo menstrual, antes da EAU e após um, três e seis meses; tempo de permanência hospitalar; sintomas imediatos pós-embolização que caracterizam a síndrome pós-embolização: náuseas ou vômitos, dor e febre decorrentes da isquemia dos nódulos de leiomioma. Durante o seguimento após a embolização foi feita a caracterização semanal da flora vaginal (bacterioscópico e cultura) nos primeiros dois meses após o tratamento. Além disto avaliamos a duração, intervalo e quantidade do sangramento menstrual antes da EAU e mensalmente após.

\section{Resultados}

A Tabela 1 relaciona os volumes do conjunto útero-leiomioma antes da EAU e três meses depois, e o respectivo decréscimo volumétrico percentual. A média dos volumes iniciais e finais foi respectivamente de 381 e $263 \mathrm{~cm}^{3}$. O maior volume uterino antes da EAU foi de $672 \mathrm{~cm}^{3}$. A redução média do volume uterino foi de $27,4 \%$, variando de $63,7 \%$ (maior redução) a casos em que a redução foi inferior a $1 \%$.

Tabela 1 - Volumes uterinos antes da embolização das artérias uterinas, 3 meses após e as respectivas variações percentuais.

\begin{tabular}{|c|c|c|c|}
\hline $\mathrm{N}^{0}$. & V1 & V2 & Redução (\%) \\
\hline 1 & 497 & 250 & 49,6 \\
\hline 2 & 273 & 99 & 63,7 \\
\hline 3 & 125 & 119 & $<1$ \\
\hline 4 & 473 & 380 & 19,6 \\
\hline 5 & 218 & 143 & 34,4 \\
\hline 6 & I.T. & I.T. & - \\
\hline 7 & I.T. & I.T. & - \\
\hline 8 & 443 & 274 & 38,1 \\
\hline 9 & 576 & 532 & $<1$ \\
\hline 10 & 525 & 372 & 29,1 \\
\hline 11 & 157 & 148 & $<1$ \\
\hline 12 & 672 & 422 & 37,2 \\
\hline 13 & 202 & 142 & 29,7 \\
\hline 14 & 440 & 405 & 7,9 \\
\hline 15 & I.T. & I.T. & - \\
\hline 16 & 233 & 185 & 20,6 \\
\hline 17 & 219 & 158 & 27,8 \\
\hline 18 & 661 & 320 & 51,5 \\
\hline Médias & 381 & 263 & 27,4 \\
\hline
\end{tabular}

V1- Volume uterino pré-embolização $\left(\mathrm{cm}^{3}\right)$

V2- Volume uterino 3 meses após a embolização $\left(\mathrm{cm}^{3}\right)$

Redução (\%) - Redução percentual

I.T.- Insucesso técnico 
Com relação às características do fluxo menstrual verificou-se a ocorrência de amenorréia ovariana em quatro pacientes, sendo que uma apresentou reversão deste quadro, caracterizando insuficiência ovariana temporária, e as outras três pacientes evoluíram para insuficiência ovariana aparentemente definitiva. O fluxo menstrual continuou aumentado após a EAU em duas pacientes, e em uma paciente houve melhora parcial dos sintomas (Tabela 2).

Tabela 2 - Evolução sintomatológica das pacientes pós-embolização das artérias uterinas (EAU).

\begin{tabular}{rll}
\hline$N^{\circ}$. & Queixas pré-EAU & Queixas pós-EAU \\
\hline 1 & HD & Amenorréia temporária \\
2 & HD & Ausentes \\
3 & HD & HD \\
4 & H & H \\
5 & HD & Amenorréia definitiva \\
6 & I.T. & I.T. \\
7 & I.T. & I.T. \\
8 & H & Amenorréia definitiva \\
9 & HD & Ausentes \\
10 & H & H \\
11 & HD & Ausentes \\
12 & H & Ausentes \\
13 & H & Ausentes \\
14 & HD & Ausentes \\
15 & I.T. & I.T. \\
16 & HD & Amenorréia definitiva \\
17 & HD & Ausentes \\
18 & HD & H \\
\hline
\end{tabular}

$\mathrm{H}$ - Excesso menstrual.

HD - Excesso menstrual e dismenorréia.

I.T.- Insucesso técnico

O tempo médio de internação foi 1,2 dias. A alta hospitalar ocorreu no dia seguinte ao procedimento em 16 pacientes. Apenas em duas pacientes a permanência foi superior a 24 horas, sendo que uma teve alta em 48 horas e a outra em 72 horas após EAU.

No primeiro dia após a EAU a dor abdominal, principal sintoma da síndrome pós-embolização, foi caracterizada como forte em $85 \%$ das pacientes. No segundo dia após o procedimento $45 \%$ das pacientes referiam persistência do quadro doloroso abdominal e no dia seguinte somente uma paciente $(5,5 \%)$ ainda apresentava dor de forte intensidade apesar da medicação analgésica instituída. A partir do $4^{\circ}$ dia nenhuma paciente referiu dor. Verificamos a ocorrência de vômitos e febre como episódios autolimitados e restritos ao primeiro dia pós-EAU em respectivamente $66 \%$ e $20 \%$ das pacientes.

O exame do conteúdo vaginal pela cultura geral e bacterioscópico foi realizado em todas as pacientes. Em três pacientes foram observadas alterações inflamatórias no exame bacterioscópico caracterizadas pela presença de numerosos leucócitos polimorfonucleares. Em uma destas pacientes foi isolada Chlamydia trachomatis, mas nas outras nenhum agente específico pôde ser demonstrado. As demais pacientes não apresentaram alterações significativas da flora vaginal. Em uma paciente ocorreu isquemia parcial do colo uterino.

\section{Discussão}

A possibilidade de tratamento definitivo sem necessidade de cirurgia abre novas perspectivas na terapêutica dos leiomiomas. $\mathrm{O}$ índice de sucesso técnico do procedimento observado por Worthington-Kirsch et al. ${ }^{8}$ é de $96 \%$. Na presente casuística ocorreram três insucessos, devido à dificuldade de acesso às artérias uterinas. A anatomia arterial da pelve feminina apresenta grande número de variações a partir da artéria ilíaca interna, fato que dificulta o acesso e a seletividade da embolização ${ }^{10}$. A taxa de $16 \%$ de insucesso técnico neste estudo está associada à curva de aprendizagem. À medida que a experiência com a EAU aumenta, esta taxa tende a diminuir.

É relatada por Goodwin et al. ${ }^{5}$, a redução, 3 meses após a EAU, de até $46 \%$ no volume do conjunto útero-leiomioma. Avaliando 80 pacientes, Ravina et al. ${ }^{6}$ verificaram a redução média de $55 \%$ no volume do maior leiomioma. Na presente casuística obtivemos redução média do volume do conjunto útero-leiomioma de $27,4 \%$, inferior ao publicado na literatura, provavelmente por tratar de amostragem inicial com menor número de pacientes em acompanhamento.

Em relação às características do fluxo menstrual, o excesso menstrual e a dismenorréia foram controlados respectivamente em $86 \%$ e $60 \%$ das pacientes. Outros autores verificaram controle dos distúrbios menstruais em até $89 \%$ das pacientes $^{6}$. Entretanto, a taxa de recorrência destes leiomiomas tratados pela EAU e a chance de aparecimento de outros nódulos ainda não são conhecidas em prazos maiores que oito anos, que é o tempo máximo de observação dos casos tratados.

A amenorréia de causa ovariana ocorre em $45 \%$ das mulheres com idade superior a $45 \operatorname{anos}^{11}$. $\mathrm{Na}$ presente casuística houve 4 casos de falência 
ovariana após a EAU, sendo que uma das pacientes, com 43 anos de idade apresentou amenorréia e elevação transitória dos níveis de gonadotropinas com subseqüente normalização, e retorno das menstruações. As outras três pacientes, com 41, 45 e 49 anos de idade evoluíram para amenorréia com insuficiência ovariana aparentemente definitiva e confirmada por meio das dosagens de FSH e LH, sem reversão do quadro até o momento atual. Em face destas alterações hormonais, ainda que eventualmente temporárias, a indicação da EAU deve ser reservada para mulheres com prole constituída e idade superior a 45 anos.

Em relação ao tempo de permanência hospitalar pós-EAU, apenas duas pacientes permaneceram mais de 24 horas por apresentarem dor, necessitando de medicação parenteral para seu controle.

A avaliação histológica de alguns úteros pósEAU revelou a presença de esclerose hialina nos leiomiomas e preservação do miométrio nor$\mathrm{mal}^{12}$. A ocorrência de endometrite ou piometrite é rara, não ultrapassando $2 \%$ dos casos ${ }^{8}$. Foi relatado um caso de óbito por septicemia pós-EAU ${ }^{13}$. Na presente casuística, a seleção e acompanhamento de pacientes pela avaliação da flora vaginal objetivou minimizar este risco. Os três casos nos quais ocorreram alterações inflamatórias foram tratados com antibióticos.

A isquemia acentuada do útero é complicação que leva à histerectomia em 1\% dos casos $^{5}$. Na presente casuística, 7 dias após o procedimento uma paciente apresentou área hipocrômica com características isquêmicas no colo uterino, provavelmente por oclusão de ramo cervical da artéria uterina. Optou-se por conduta expectante com antibioticoterapia sistêmica, constatando-se regressão completa do quadro em 45 dias. Essa ocorrência não foi relatada na literatura até o momento.

Algumas dúvidas em relação aos riscos da EAU devem ser discutidas. Assim, em relação ao risco de malignidade dos leiomiomas, apenas 0,4\% das pacientes que são submetidas a histerectomia por diagnóstico de leiomiomatose uterina apresentam exame anatomopatológico compativel com câncer ${ }^{14}$. Não existem relatos a respeito do aparecimento de tumores malignos pós-EAU.

Outra preocupação diz respeito à exposição à radiação. A dose necessária para a EAU é $10 \mathrm{a}$ 30 vezes maior do que aquela usada em exames radiográficos convencionais, porém também é de 10 a 30 vezes menor que a utilizada em radioterapia para a doença de Hodgkin. Os autores ressaltam que pacientes com doença de Hodgkin submetidas à radioterapia não mostraram maior incidência de infertilidade ou defeitos genéticos ${ }^{15}$.
Concluímos que a EAU representa nova alternativa terapêutica para o tratamento dos leiomiomas, entretanto são necessários estudos prospectivos para avaliar a eficácia e os riscos do procedimento. Tendo em vista o risco de insuficiência ovariana este procedimento deve ser reservado inicialmente para pacientes acima dos 45 anos de idade ou com prole constituída.

\section{SUMMARY}

Purpose: to evaluate the results of uterine artery embolization $(U A E)$ in the treatment of uterine leiomyomas.

Methods: eighteen patients with ultrasonographic diagnosis of uterine leiomyomas were submitted to UAE with polyvinyl alcohol (PVA) particles. The femoral arteries are the access sites until selective catheterization of the uterine arteries. Imaging regarding uterine volume was performed before the procedure and three months after wards. Clinical follow-up was performed at regular intervals after the procedure to assess patient menstrual characteristics and uterine volume. Results: three procedures were technically unsuccessful because of failure of superselective catheterization. Control of menorrhagia and pelvic pain was reported at three months after the procedure by 86 and $60 \%$ of patients, respectively. The initial mean uterine volume was $381 \mathrm{~cm}^{3}$ and after 3 months, $263 \mathrm{~cm}^{3}$. The mean uterine volume reduction was $27.4 \%$ after three months of follow-up. Clinical and biochemical findings consistent with ovarian failure were observed in three (20\%) patients.

Conclusions: UAE represents a new therapeutic approach in the treatment of uterine leiomyomas. The risk of ovarian failure after the procedure limits its use to patients aged 45 years or without pregnancy wish.

KEY WORDS: Uterine leiomyomas. Embolization, uterine artery. Ovarian failure.

\section{Referências}

1. Bozzini N, Liao A, Pereira PA et al. Análogos do GnRH no tratamento do leiomioma uterino. Femina 1997; 25:701-4.

2. Ribeiro SC, Reich H, Rosenberg J, Guglielminetti E, Vidali A. Laparoscopic myomectomy and pregnancy outcome in infertile patients. Fertil Steril 1999; 71:571-4.

3. Duarte G. Doenças benignas do corpo uterino. In: Halbe HW, editor. Tratado de Ginecologia. $2^{\mathrm{a}}$ ed. São Paulo: Roca; 1993. p.1073-95. 
4. Ravina JH, Herbreteau D, Ciraru-Vigneron N, et al. Arterial embolization to treat uterine myomata. Lancet 1995; 346:671-2.

5. Goodwin S, Vedantham S, McLucas B, Forno AE, Perrella R. Preliminary experience with uterine artery embolization for uterine fibroids. J Vasc Interv Radiol 1997; 8:517-26.

6. Ravina JH, Bouret JM, Ciraru-Vigneron N, et al. Application of particulate arterial embolization in the treatment of uterine fibromyomata. Bull Acad Natl Med 1997; 181:233-46.

7. Bradley EA, Reidy JF, Forman RG, Jarosz J, Baude PR. Transcatheter uterine artery embolization to treat large uterine fibroids. $\mathrm{Br} \mathrm{J}$ Obstet Gynaecol 1998; 105:235-40.

8. Worthington-Kirsch RL, Popky GL, Hutchins FL. Uterine arterial embolization for the management of leiomyomas: quality-of-life assessment and clinical response. Radiology 1998; 208:625-9.

9. Seldinger S.I. Catheter replacement of needle in percutaneous arteriography. A new technique. Acta Radiol 1953; 39:368-76.
10.Pelage JP, Le Dref O, Soyer P, et al. Arterial anatomy of the female genital tract: variations and relevance to transcatheter embolization of the uterus. AJR Am J Roentgenol 1999; 172:989-94.

11.Chrisman HB, Saker MB, Ryu RK, et al. The impact of uterine fibroid embolization on resumption of menses and ovarian function. J Vasc Interv Radiol 2000; 11:699-703.

12.Siskin GP, Eaton LA, Stainknen BF, Dowling K, Herr A, Schwartz J. Pathologic findings in a uterine leiomyoma after bilateral artery embolization. J Vasc Interv Radiol 1999; 10:891-4.

13.Walker W, Worthington-Kirsch RL. Fatal septicaemia after fibroid embolisation. Lancet 1999; 354:1730-3.

14.Takamizawa S, Minakami H, Usui R, et al. Risk of complications and uterine malignancies in women undergoing hysterectomy for presumed benign leiomyomas. Gynecol Obstet Invest 1999; 48:193-6.

15.Nikolic B, Spies JB, Lundsten MJ, Abbara S. Patient radiation dose associated with uterine artery embolization. Radiology 2000; 214:121-5.

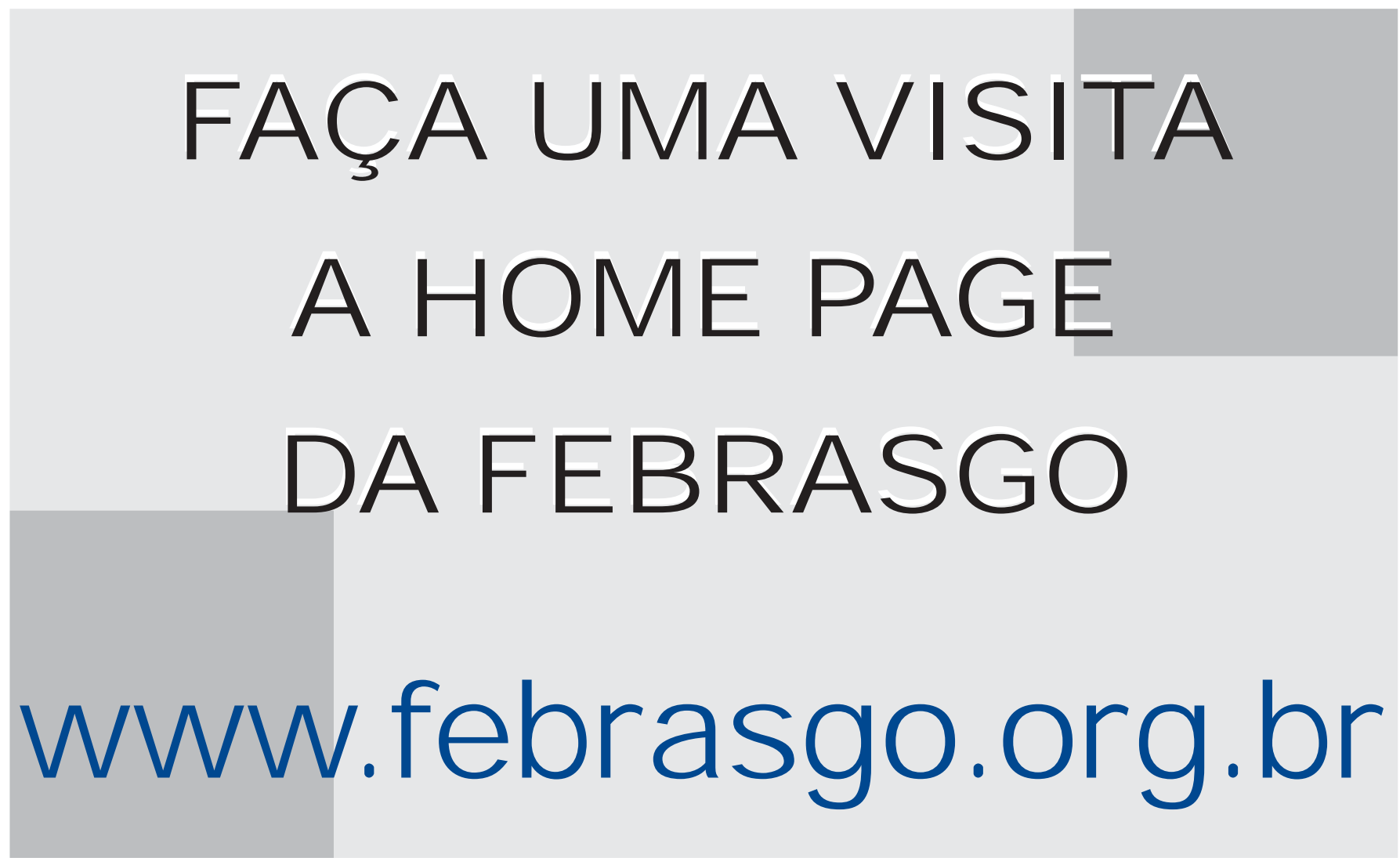

